\title{
P02-023 - NLRP3 mosaicism as a cause of late-onset CAPS
}

\author{
Q Zhou*, AK Ombrello, D Chin, DL Kastner, I Aksentijevich \\ From 7th Congress of International Society of Systemic Auto-Inflammatory Diseases (ISSAID) \\ Lausanne, Switerland. 22-26 May 2013
}

\begin{abstract}
Introduction
The dominantly inherited cryopyrin-associated periodic syndromes (CAPS) are caused by heterozygous missense gain-of-function mutations in the NLRP3 (CIAS1) gene encoding NLRP3 (also known as cryopyrin). Most patients present at a young age with a variety of clinical symptoms including fevers, urticaria-like skin rash, arthropathy, and CNS inflammation. A subset of patients followed at the National Institute of Health's autoinflammatory disease clinic has adult-onset fevers and urticarial rash but conventional genetic testing has been unremarkable for any mutations in NLRP3. We analyzed one such "mutationnegative" patient. She is a 63y/o female of Irish ancestry who developed a gradually worsening stress-induced urticarial rash in her 40s. Additional clinical history is remarkable for severe arthralgia, myalgia, chills, and occasional conjunctivitis. Initially started on anakinra $100 \mathrm{mg} /$ day in 2003, she had dramatic improvement in symptoms; however, her anakinra dose has required periodic adjustments since that time to control her symptoms.
\end{abstract}

\section{Objectives}

To identify the cause of disease in a patient who presented with late-onset CAPS and who was mutation-negative in NLRP3 based on the conventional Sanger sequencing.

\section{Methods}

We performed whole exome sequencing in the DNA sample extracted from peripheral blood. A total of 192 subclones were randomly selected and subjected to Sanger sequencing to validate the NLRP3 somatic mutation. Targeted deep sequencing on NLRP3 will be done using the DNA from blood and buccal cells.

\section{Results}

Our established exome data analysis pipeline failed to identify any plausible candidate gene in this patient. In order to increase the sensitivity of variant calling, we used a different combination of parameters including calling SNPs/Indels with extreme strand bias, which resulted in a rapid increase in false positive rate but also raised the possibility of identifying somatic mosaicism mutations. Based on this new analytic approach, we identified a possible mutation p.Tyr570Cys (c.1709A >G) in NLRP3. Exome data showed that there are 12 reads carrying the mutant $\mathrm{G}$ allele and 64 reads carrying the wild type A allele (15\%). This mutation has been previously reported in a NOMID/ CINCA patient from Australia. The putative mosaic p. Tyr570Cys mutation was validated by subcloning a 718bp fragment from the patient's leukocyte DNA and subsequent Sanger sequencing of 192 clones. We found 20 clones carrying the p.Tyr570Cys mutation, which established the level of somatic mosaicism at $10.4 \%$. We are in the process of evaluating this mosaic mutation in other tissues from this patient.

\section{Conclusion}

We report NLRP3 somatic mosaicism in a patient presenting with a late onset CAPS. To our knowledge this is the first time that a NLRP3 mutation has been identified in patients developing symptoms in adulthood. Other possible causal genes have been ruled out based on exome sequencing analysis. Our result contributes significantly to understanding the pathogenesis of disease in patients with adult-onset autoinflammatory diseases.

\section{Disclosure of interest}

None declared. 
- Convenient online submission

- Thorough peer review

- No space constraints or color figure charges

- Immediate publication on acceptance

- Inclusion in PubMed, CAS, Scopus and Google Scholar

- Research which is freely available for redistribution 